\title{
Next generation sequencing in gastric or gastroesophageal adenocarcinoma
}

\author{
Geoffrey Y. Ku \\ Gastrointestinal Oncology Service, Department of Medicine, Memorial Sloan Kettering Cancer Center, New York, NY, USA \\ Correspondence to: Geoffrey Y. Ku, MD. 300 E. 66th Street, Rm 1035, New York, NY 10065, USA. Email: kug@mskcc.org.
}

\begin{abstract}
Seminal analyses of The Cancer Genome Atlas (TGCA) and Asian Cancer Research Group (ACRG) have provided unprecedented insight into the molecular underpinnings of gastric cancer (GC). At the same time, next generation sequencing (NGS) panels, driven by quantum improvements in DNA sequencing technology and bioinformatics, are now routinely used in standard clinical care and also extensively for research purposes. This review article will discuss the molecular subtypes of GC, the current standard-of-care therapies for GC and the role of NGS in standard care and in research.
\end{abstract}

Keywords: Next generation sequencing (NGS); gastric cancer (GC); targeted therapy

Received: 21 November 2019; Accepted: 15 January 2020; Published: 05 October 2020.

doi: 10.21037/tgh.2020.01.09

View this article at: http://dx.doi.org/10.21037/tgh.2020.01.09

\section{Introduction}

Gastric cancer (GC), an uncommon but highly virulent malignancy in the United States, will be diagnosed in 27,510 patients in 2019, with 11,140 deaths (1). In comparison to its relative rarity in the U.S., GC is endemic in parts of East Asia, which account for more than half of the approximately one million cases that develop per year globally (2). Despite the much higher incidence, East Asian patients with GC do appear to have a better prognosis (3).

In the U.S., the incidence of GC has decreased significantly in the past 50 years but the location of the primary tumor has also changed. Distal GC, which previously predominated, has become uncommon, while the incidence of tumors of the gastric cardia and gastroesophageal junction (GEJ) have increased $4 \%$ to $10 \%$ per year among U.S. men since $1976(4,5)$.

Changing epidemiologic factors account for the increasing incidence of proximal tumors. Chronic infection with Helicobacter pylori has been implicated in the development of GC on the basis of epidemiological evidence (6). A decline in $H$. pylori infection in the U.S. has led to an overall decrease in the number of GC cases. On the other hand, proximal and GEJ tumors are now more common because of an increased incidence of gastroesophageal reflux disease (7) and obesity (8).

Given the clinical heterogeneity of GC on a global basis, there have been intensive efforts to better understand the molecular basis of GC. These efforts culminated in the analyses first by The Cancer Genome Atlas (TGCA) Network (9) and then by the Asian Cancer Research Group (ACRG) (10) that have for the first time defined distinct molecular subtypes of GC.

In parallel and perhaps accelerated by the results of the TCGA and ACRG analyses, it is now considered standardof-care and reimbursed by insurance for U.S. patients with GC to undergo next generation sequencing (NGS) of their tumors either through commercial or academic (e.g., MSK-IMPACT) platforms, ostensibly under the rubric of "personalized medicine" in order to identify actionable alterations that form the basis of targeted therapies.

In this review article, I will discuss the molecular subtypes identified by the TCGA and ACRG groups, discuss the current standard-of-care for the treatment of GC and then focus on experimental applications and the standard clinical utility of NGS.

\section{Molecular profiles of GC}

In 2014, TCGA network presented their landmark analysis, 
where they characterized 295 localized previously untreated gastric adenocarcinomas based on six molecular platforms: somatic copy number analysis, whole-exome sequencing, DNA methylation profiling, messenger RNA sequencing, microRNA sequencing and reverse-phase protein array (9). In addition, microsatellite instability (MSI) testing and whole-genome sequencing were performed. Mindful of the geographic variation in the clinicopathologic characteristics of GC, the tumor samples came from North America, Western and Eastern Europe and East and Southeast Asia.

TCGA analyses identified four GC subtypes: (I) tumors positive for Epstein-Barr virus (EBV) (9\%); (II) MSI-high (termed MSI by TCGA analysis) tumors (22\%); (III) genomically stable (GS) tumors (20\%) and; (IV) tumors with chromosomal instability (CIN) $(50 \%)$. Each of these subgroups had distinct molecular characteristics: EBV-positive tumors exhibit high levels of DNA hypermethylation, recurrent PIK3CA and ARIDIA mutations and amplification of the genes encoding the programed death ligand-1 (PD-L1) and PD-L2 proteins.

MSI tumors are associated with hypermethylation of the MLH1 gene and exhibit elevated mutation rates, including mutations of genes encoding targetable oncogenic signaling proteins. Interestingly, MSI tumors occurred in older patients (median age 72) who were predominantly women (56\%).

GS tumors are more commonly found in the diffuse histology and carry mutations of CDH1 and RHOA, as well as a CLDN18-ARHGAP26 fusion. The RHOA protein is implicated in actin-myosin-dependent cell contractility and cellular motility and activates STAT3 to promote tumorigenesis. As such, modulation of RHOA may contribute to the disparate growth patterns and lack of cellular cohesion that are hallmarks of diffuse tumors. CLDN18 is a component of the tight junction adhesion structures. As such, these fusions may disrupt wild-type CLDN18, impacting cellular adhesion. Interestingly, RHOA mutations and the CLDN18-ARHGAP26 fusions were mutually exclusive.

Finally, CIN tumors are frequently observed at the GEJ/ cardia and are mostly intestinal-type cancers, with recurrent TP53 mutation and relatively numerous amplifications of receptor tyrosine kinase $(R T K)$ genes, as well as the gene encoding vascular endothelial growth factor (VEGF)-A.

In 2015, the ACRG also proposed four molecular subtypes based on evaluation of 251 GCs from Korea, including: (I) MSI-H; (II) microsatellite stable (MSS) with epithelial-mesenchymal transition features (MSS/EMT);
(III) MSS/TP53 active (MSS/TP53 ${ }^{+}$and; (IV) MSS/TP53 inactive (MSS/TP53 ${ }^{-}$) (10).

These subtypes are associated with specific clinicopathologic features. The MSI subtype is associated with hypermutation, occurs predominantly in the antrum, is associated with intestinal histology, is more likely to be diagnosed at an early stage and has the best prognosis.

The MSS/TP53 ${ }^{+}$and MSS/TP53- subtypes have an intermediate prognosis. Tumors of the MSS/TP $53^{+}$subtype have the highest incidence of EBV positivity and have a high prevalence of mutations in the APC, KRAS, PIK3CA, $A R I D 1 A$ and SMAD4 genes compared to the MSS/TP53 ${ }^{-}$ subtype. In comparison, the MSS/TP53- subtype-true to its name-has the highest prevalence of TP53 mutations, with a low frequency of mutations in other genes.

Finally, the MSS/EMT subtype conveys the worst prognosis; these tumors are associated with a high rate of recurrence and the highest risk of peritoneal carcinomatosis. It predominantly consists of diffuse tumors and tends to be found in patients diagnosed at a younger age. This subtype has low cell adhesion due to loss of $C D H 1$ and has the least number of mutations. ARID1A is among the most frequently mutated gene.

The differences between the two classifications (TGCA and ACRG) reflect the different approaches and platforms used, and the ethnicity of the samples (global vs. Korean). However, similarities do exist. Both analyses identified an MSI subtype with hypermethylation of MHL1, hypermutation and the most favorable prognosis. The EBV and MSS/TP53 ${ }^{+}$subtypes are similar in that both subtypes have the highest prevalence of EBV infection and carry mutations in PIK3CA and ARID1A. The GS and MSS/ EMT subtypes, which include younger patients, are mostly diffuse tumors and show low intercellular adhesion. The CIN and MSS/TP53- subtypes are mostly intestinal tumors and carry mutations in TP53.

Both of these complementary analyses are groundbreaking. For the first time, they allow for the accurate molecular characterization of GC that uses a common language and moves beyond the century-long limitation of description based primarily on location (GEJ/proximal vs. mid $v s$. distal) and histology (diffuse $v s$. intestinal, amongst other classification systems). The potential to better identify patients for experimental strategies, the ability to accurately compare patients across different clinical studies and the opportunity to use these molecular subtypes as springboards for drug discovery are truly unprecedented.

Nevertheless, it is important to note that both 
analyses required massive and complex laboratory research methodologies that cannot be recapitulated in standard clinical care. Therefore, a major and heretofore unsurmountable challenge remains the approximation of these molecular subtypes in real-time in the clinic or even post hoc in the context of a well-funded research study, where ample quantities of tumor and matched normal tissue or blood samples, as well as meticulously annotated clinical data, are not always readily available.

\section{Standard-of-care therapy for GC}

\section{Metastatic disease: first-line therapy}

Globally, the standard regimen in the metastatic setting consists of a fluoropyrimidine/platinum doublet. In the U.S., most oncologists treat patients with the FOLFOX regimen (bolus and infusional 5-fluorouracil or 5-FU/leucovorin/ oxaliplatin) $(11,12)$.

At this time, the only validated targeted therapy in the first-line setting is trastuzumab, an anti-Her2 antibody, which is added to chemotherapy for the $20-25 \%$ of GCs that over-express the Her2 protein (13). Current guidelines for the assessment of Her2 positivity call for initial testing by immunohistochemistry (IHC) and fluorescent in situ hybridization (FISH) only for tumors that are intermediate by IHC (score 2+) (14). IHC 0/1+ and IHC $2+/$ FISH negative tumors are considered Her 2 negative, while IHC $3+$ and IHC $2+/$ FISH positive tumors are Her 2 positive. Of note, Her2 staining in GC is considerably more heterogenous than in breast cancer such that any tumor where $\geq 10 \%$ of cells demonstrate strong staining are considered IHC $3+$. As one can imagine, that leads to significant variation in positivity for tumors that are all broadly considered to be Her2 positive.

\section{Metastatic disease: anti-angiogenic therapy}

Since 2014, the global standard-of-care in the second-line setting has been ramucirumab, an anti-VEGF receptor antibody, in combination with paclitaxel chemotherapy (15). The phase III RAINBOW study showed clear improvement in all outcomes for this combination $v s$. single-agent paclitaxel.

In China, apatinib, an anti-VEGFR tyrosine kinase inhibitor, has been approved as third-line therapy based on a positive phase III study vs. placebo (16). However, this drug is not available in the U.S.

\section{Metastatic disease: immunotherapy}

Immune checkpoint inhibitors (ICIs) have a clearly defined role in treatment-refractory GC, although the benefit of these drugs is modest. In the U.S., pembrolizumab is approved as third-line or greater therapy for patients whose tumors are $\mathrm{PD}-\mathrm{L} 1$ positive by the combined positive score $(\geq 1)$ using the $22 \mathrm{C} 3$ antibody, based on a single arm of the KEYNOTE-059 study (17). In Japan, nivolumab is approved in a similar setting but irrespective of PD-L1 status, based on the phase III ATTRACTION-2 study (18). Both studies revealed essentially identical results for these anti-PD-1 antibodies.

Efforts to move these drugs into the first- and secondline setting have unfortunately failed. The KEYNOTE-061 study did not show superiority of pembrolizumab vs. paclitaxel in the second-line setting (19) and the recently presented KEYNOTE-062 also failed to demonstrate superiority of pembrolizumab and chemotherapy $v s$. chemotherapy alone; the study did suggest that pembrolizumab is non-inferior to chemotherapy, although response rates and progression-free survival (PFS) were significantly inferior for pembrolizumab (20).

The only other FDA-approved indication for pembrolizumab is in the second-line setting for any MSI cancer, based on a pivotal single-arm study that established remarkable activity for ICIs in these hypermutated tumors (21). In GC, MSI tumors occur in about 3-4\% of patients with metastatic disease $(17,19)$. This is markedly lower than the $22 \%$ prevalence of the MSI subtype in TCGA analysis, a difference that may be partially explained by the fact that the analysis involved localized, non-metastatic cancers; the incidence of MSI tumors in the localized setting is higher (about 7\%; see below), presumably because these cancers have a favorable prognosis and relatively low risk of disease recurrence.

\section{Locally advanced disease: peri-operative chemotherapy}

In the U.S. and Western Europe, the current standard is pre-operative chemotherapy with the FLOT (5-FU/ leucovorin/oxaliplatin/docetaxel) regimen, based on the phase III FLOT4 study (22). In East Asia, the standard is upfront surgery and adjuvant chemotherapy, with validated options including a fluoropyrimidine with oxaliplatin [Korean CLASSIC study (23)] or with docetaxel [Japanese JACCRO GC-07 study (24)].

Recently, a retrospective analysis from the MAGIC 
study strongly suggests that patients with mismatch repair protein deficient (dMMR) or MSI tumors are harmed by pre-operative chemotherapy (25). For patients who undergo surgery alone, those with $\mathrm{d} M M \mathrm{MR} / \mathrm{MSI}$ tumors $(6.7 \%$ of the cohort) had improved outcomes compared to those who were MSS. Furthermore, a recent post-hoc analysis of the CLASSIC study identified that $6.8 \%$ of patients were MSI (26). These patients had improved survival vs. MSS patients and did not benefit from adjuvant chemotherapy. These results have now been confirmed in an individual patient meta-analysis of these two studies, as well as the ARTIST and ITACA-S studies of adjuvant treatment (27). In this analysis, 5-year disease-free survival and overall survival were not statistically different for MSI patients who did and did not receive chemotherapy, although the point estimate was lower for those who received chemotherapy.

\section{Standard biomarker analyses}

Based on the approaches above, the following biomarkers are considered standard for U.S. patients with metastatic GC: Her2 (by IHC and/or FISH), MMR testing by IHC or MSI testing by PCR and PD-L1 assessment by IHC (using the $22 \mathrm{C} 3$ or other validated antibody). In the locally advanced setting, we routinely perform MMR or MSI testing as patients with $\mathrm{dMMR} / \mathrm{MSI}$ GCs are referred for upfront surgery instead of pre-operative chemotherapy.

At this time, NGS does not play a role in standard clinical care.

\section{NGS panels}

In the past decade, there has been an exponential increase in the availability and use of NGS platforms in cancer through the development of massively parallel NGS technologyitself a culmination of improvements in DNA sequencing technology, computer power and bioinformatics-that allows for the rapid sequencing of a panel of 300-500 selected cancer genes. In addition to providing information about genetic alterations, most NGS panels can also provide an assessment of the MSI status and tumor mutation burden (TMB) of tumors. Testing can be performed on fresh frozen paraffin embedded tissue, with a turnaround time of several weeks. In the U.S., there are now numerous commercial companies that provide NGS testing for any tumor type. Many academic institutions have also developed their own panels.

At Memorial Sloan Kettering Cancer Center, patients undergo testing with MSK-IMPACT, a 468-gene panel, which provides information about single nucleotide variants, insertions and deletions (28). MSI status is assessed by MSIsensor, a bioinformatics tool that strongly correlates with standard methodologies for MSI assessment (29). Unlike commercial NGS panels, MSK-IMPACT also requires the collection of whole blood so that genomic DNA can be extracted to serve as a matched normal control. This allows for somatic $v s$. germline mutations to be distinguished, which offers critical information in terms of counseling and potential screening strategies for patients with germline mutations in known cancer susceptibility genes. Finally, the incidental extraction of DNA from leukocytes in the blood samples has led to the additional ability to detect clonal hematopoiesis (30).

\section{Experimental roles for NGS}

\section{Identify actionable alterations to guide therapy}

Possibly the chief consideration of a clinician who orders an NGS panel is to identify an actionable alteration, for which we can consider either enrollment onto a genotypematched study or to permit the use of a currently available treatment as an "off-label" indication. In this regard, the use of NGS in advanced GC is considered a Category 2A recommendation by the National Comprehensive Cancer Network guidelines "for the identification of treatment and/ or clinical trial enrollment." They do recommend NGS be performed "in patients with advanced cancer in later stages of therapy rather than in the early phases of disease".

Unfortunately, NGS expands treatment options for patients much less frequently than hoped for. In a review of the experience at MD Anderson Cancer Center from 2015, Meric-Bernstam and colleagues noted that, while 39\% of 2,000 patients with different solid tumors who underwent NGS had at least one actionable alteration, only $4 \%$ of this cohort actually received treatment on a genotypematched study (31). How many of these patients had GC is not known and information about their outcomes was not provided so there is no way to ascertain the clinical benefit of these experimental therapies. The authors cited various challenges to trial accrual even in patients with clearly actionable alterations, including patient preference for standard treatment or treatment locally, poor performance status, lack of trials/slots and insurance denial.

As this study dates from 2015 and does not specifically separate out patients with gastrointestinal cancers, one 
may argue that it is not relevant to the present situation, where the armamentarium of experimental treatments has (slightly) expanded. However, our contemporary experience at MSKCC in enrolling patients with esophagogastric cancer (EGC) onto genotype-matched studies is similarly poor. In a 2019 abstract presentation, we noted that, out of 137 patients who had initiated first-line chemotherapy at MSKCC and who had undergone MSK-IMPACT testing, none went onto a genotype-matched study based on its results (32). These data are being updated for publication and the reasons for the lack of trial enrollment will be explored in more detail.

At a minimum, these findings should prompt clinicians to restrict the use of NGS to patients with good performance status and who are being treated at or who are in close proximity to a comprehensive cancer center with a large phase I trial portfolio. Nationally, there are also genotypematched studies, e.g., NCI's MATCH study, that are open to accrual in multiple centers.

\section{Identify biomarkers of response/resistance to current therapies}

Another major research objective of NGS is to try to shed light on inherent or acquired mechanisms of response or resistance to current therapies that go beyond the standard biomarkers. In this regard, NGS has proven to be of significant value.

\section{Anti-Her2 therapy}

Our group sequenced the tumor of 68 patients with Her2 positive EGC with MSK-IMPACT (33). We observed a concordance rate of $93.7 \%$ between IHC/FISH and NGS. A total of 50 patients with Her2-positive tumors collected before treatment received first-line trastuzumab-based therapy. Of these, $92 \%(46 / 50)$ were ERBB2-amplified by NGS. Detailed analysis of the four discordant patients indicated that the discordance was attributed to either tumor heterogeneity for ERBB2 amplification or equivocal IHC/FISH positivity. Additionally, the four patients with discordant cases exhibited significantly shorter PFS on first-line trastuzumab/chemotherapy compared to patients with ERBB2 amplified tumors by NGS (median PFS 5.8 vs. 14.0 months, $\mathrm{P}=1 \times 10^{-6}$ ). Essentially, Her2 staining is heterogenous and the more methodologies that show a tumor is Her2 positive, the more homogenous and positive Her2 expression is.

Beyond ERBB2 itself, we observed significant heterogeneity in the pattern of co-mutational events in the Her2-positive cohort. Patients with co-alterations in RTK-RAS-PI3K/AKT pathway genes had significantly shorter PFS, suggesting that activation of this pathway may contribute to intrinsic trastuzumab resistance. In a multivariate analysis, ERBB2 levels of amplification and coalterations in the PI3K pathway independently contributed to the differences in PFS.

Finally, 23 patients had NGS performed at baseline and upon progression on trastuzumab-based therapy. Notable mechanisms of resistance included the loss of $E R B B 2$ amplification in $16 \%$ of post-progression samples (confirmed by IHC), a focal ERBB2 exon 16 deletion which confers resistance to anti-Her2 therapies and the appearance of $K$-ras and PIK3CA mutations. Identification of these mechanisms of resistance is of paramount importance and allows us to begin to develop potential strategies to overcome them.

\section{Anti-VEGF therapy}

Despite intensive efforts since the advent of anti-angiogenic therapy, no biomarker has ever been identified. Studies of ramucirumab have not found a correlation in baseline angiogenic factors with outcomes (34). Biomarker analysis of the negative phase III AVAGAST study of bevacizumab, the VEGF antibody, and chemotherapy suggested that higher baseline VEGF-A and lower levels of neuropilin-1, a transmembrane glycoprotein that is a coreceptor for the VEGF family, were associated with a trend toward benefit for bevacizumab (35); however, these results have never been replicated in other data sets.

To my knowledge, NGS of tumor tissue has never been used to try to identify biomarkers for any anti-VEGF pathway therapy.

\section{ICIs}

Our group recently evaluated the molecular correlates of response to ICIs in 161 patients with advanced EGC, 89 of whom had NGS performed by MSK-IMPACT (36).

The median TMB was 5.6 (interquartile range, 3.3-8.8). In PD-L1-positive patients, the median TMB was 6.1 (range, 1.1-62) while in PD-L1-negative patients the median TMB was 3.7 (range, 1.7-9.8); $\mathrm{P}=0.038$. For each 1 unit increase of TMB, there was an association with improved PFS (HR 0.97; 95\% CI, 0.95-0.99; P=0.003) and OS (HR 0.72; 95\% CI, 0.55-0.94; $\mathrm{P}=0.016$ ), although this was not maintained in multivariate analysis. Furthermore, when MSI patients $(n=12)$ were excluded from the TMB 
analysis, we observed no significant difference in PFS or OS per 1 unit increase in TMB. Although these numbers are relatively small, they suggest that TMB is not a predictive biomarker of response to ICI in MSS EGC.

Similarly, we did not identify any genetic alteration on NGS that correlated with survival outcomes to ICI.

\section{Identify biomarkers of response to novel therapies}

Anti-epidermal growth factor receptor (EGFR) therapy Anti-EGFR therapy would seem to be an odd addition to the list of novel therapies, given two phase III studies [the EXPAND (37) and REAL-3 (38) trials] that showed no benefit and even the possibility of harm for adding an antiEGFR antibody to first-line chemotherapy in the metastatic setting. Neither study identified a biomarker of response nor their results helped to collectively kill any further evaluation of anti-EGFR therapies in this disease.

Recently, Maron and colleagues from the University of Chicago treated 7 patients who were identified on NGS to have EGFR-amplified tumors (5\% prevalence in their cohort) with anti-EGFR antibodies (with or without chemotherapy) (39). The response rate was $58 \%$ (5 of 7 patients) and all patients had disease control, with a median PFS of 10 months. These investigators also identified potential intrinsic and acquired mechanisms of resistance, again highlighting another experimental role for serial NGS.

These intriguing data suggest that there might be a rationale to revisit anti-EGFR antibodies in a biomarker selected population based on NGS.

\section{PARP inhibitors}

Both TCGA and ACRG analyses suggest that mutations in genes involved in homologous DNA repair, including $B R C A 1 / B R C A 2$ and $A T M$, are relatively common. It has been hypothesized that such tumor cells are particularly sensitive to treatment with poly (ADP-ribose) polymerase (PARP) inhibitors, which inhibit this important component of the base excision repair pathway. Proof of this concept in a gastrointestinal cancer came from a recent phase III study of maintenance therapy with olaparib following initial treatment with a platinum-based regimen in germline $B R C A-1 / 2$-mutated pancreas cancer (40).

In GC, completed randomized studies have unfortunately been negative. Olaparib was initially evaluated in a randomized phase II study of paclitaxel alone or with the combination; the study was specifically enriched for tumors that had low expressions of the ATM protein to $50 \%$ of the enrolled patients (41). Olaparib/paclitaxel significantly improved OS vs. placebo/paclitaxel in both the overall population ( $\mathrm{HR}, 0.56 ; \mathrm{P}=0.005)$ and the $\mathrm{ATM}_{\text {low }}$ population (HR, 0.35; $\mathrm{P}=0.002$ ).

These results paved the way for the phase III GOLD study (42); unlike the previous study, there was no attempt to enrich for patients with $\mathrm{ATM}_{\text {low }}$ tumors so that only $18 \%$ of tumors ( $<50$ patients in each arm) were ultimately found to be $\mathrm{ATM}_{\text {low }}$ based on pre-planned analyses performed following enrollment. Olaparib did not improve survival in either the intention-to-treat (ITT) or ATM $\mathrm{Amw}_{\text {low }}$ population, although the HR of 0.79 in the ITT population approached statistical significance $(\mathrm{P}=0.026)$.

The negative results of the GOLD study potentially argue that ATM protein expression is not a useful biomarker, although the very small number of $\mathrm{ATM}_{\text {low }}$ patients may have limited the statistical power. In addition, the study had 2 co-primary endpoints-OS improvement in the ITT and $\mathrm{ATM}_{\text {low }}$ populations-which resulted in a statistical significance threshold of $\mathrm{P}<0.025$ that was barely missed. The study was also designed based on the relatively audacious HRs observed in the randomized phase II study, which may have rendered it underpowered to detect a smaller benefit.

Despite these results, there does appear to be a signal of activity to continue to evaluate PARP inhibitors in this disease. The use of NGS to identify tumors with mutations in the homologous recombination deficiency pathwaywhich include BRCA1/2, ATM, CHEK1/2 and PALB2could therefore serve as a future tool to select patients for such studies. In addition, a study by our group in pancreas cancer suggests that patients with progression on a platinum-based regimen are very unlikely to benefit from PARP inhibition (43) and this clinical feature can also be used as part of the selection strategy in GC.

\section{Anti-fibroblast growth factor receptor (FGFR)-2 therapy}

FGFR2 amplification occurs in 5-10\% of GCs. The SHINE study was a randomized phase II study that enrolled patients with $F G F R 2$ polysomy or gene amplification detected by FISH and randomized them to receive AZ4547, a selective FGFR-1,2,3 TKI vs. paclitaxel (44). Median PFS was not improved by the experimental drug. Biomarker analyses noted considerable intratumor heterogeneity for FGFR2 gene amplification and poor concordance between FGFR2 amplification/polysomy and FGFR2 expression, suggesting that FISH may have been a suboptiomal biomarker to select 
patients for this study.

Other FGFR TKIs show some early promise in EGC. Results for TAS-120 have been presented in abstract form and revealed partial responses in 2 patients with EGC, one of whom was known to have an FGFR2 amplified tumor (45). Another drug is bemarituzumab, a monoclonal antibody against FGFR2b receptor; it has modified to enhance antibody-dependent cell-mediated cytotoxicity. It is undergoing phase III evaluation in the FIGHT study, where it is added to FOLFOX chemotherapy for patients whose tumors either have FGFR2b protein overexpression by IHC or who have FGFR2 gene amplification detected by circulating tumor DNA (ctDNA).

Therefore, for these and other studies, NGS may be useful to detect these uncommon alterations in the FGFR pathway to select patients.

\section{ctDNA}

Key limitations of NGS of tumor tissue include the heterogeneity within the same tumor and within different tumors at different sites and changes with time and through different therapies. Repeat biopsies or biopsies of several sites are generally infeasible. As such, ctDNA analysis through so-called "liquid biopsies" has rapidly emerged as a promising technique that can overcome many of the logistic challenges of frequent sampling and can also address issues of tumor heterogeneity (46). NGS methods have been modified for ctDNA, ranging from whole-genome or whole exome sequencing to targeted sequencing of a limited gene panel. Studies in multiple tumor types have shown good concordance between the results of tumor NGS and ctDNA analysis.

In conjunction with tumor-based NGS, ctDNA analysis may also be used for most of the experimental purposes discussed above. In addition, ctDNA has been proposed for early detection of cancers, to monitor minimal residual disease in patients who have completed definitive therapy and surgery, to detect early recurrence and to monitor response to therapies.

\section{Conclusions}

The use of NGS has now become increasingly routine in GC. Despite its common usage, there are actually no standard-of-care applications and it rarely expands experimental treatment options based on currently available therapeutics. Nevertheless, there are multiple research applications for NGS, including the identification of markers of response/resistance to known therapies and the identification of uncommon molecular subsets of patients that may benefit from emerging therapies. Finally, an adjunct technology—ctDNA—will likely place an increasing role not only in these experimental indications but also in emerging areas, such as the early detection/screening of cancer, the early detection of recurrence and the ability to obtain an aggregate assessment of tumor heterogeneity at multiple time-points.

\section{Acknowledgments}

Funding: None.

\section{Footnote}

Conflicts of Interest: Arog Pharmaceuticals-research support; AstraZeneca-research support, consulting; Bristol-Myers Squibb-research support, consulting; Daiichi Sankyoresearch support; Eli Lilly—consulting; Merck—research support, consulting; Pieris Pharmaceuticals—research support, consulting; Zymeworks—research support.

Ethical Statement: The author is accountable for all aspects of the work in ensuring that questions related to the accuracy or integrity of any part of the work are appropriately investigated and resolved.

Open Access Statement: This is an Open Access article distributed in accordance with the Creative Commons Attribution-NonCommercial-NoDerivs 4.0 International License (CC BY-NC-ND 4.0), which permits the noncommercial replication and distribution of the article with the strict proviso that no changes or edits are made and the original work is properly cited (including links to both the formal publication through the relevant DOI and the license). See: https://creativecommons.org/licenses/by-nc$\mathrm{nd} / 4.0 \%$.

\section{References}

1. Siegel RL, Miller KD, Jemal A. Cancer statistics, 2019. CA Cancer J Clin 2019;69:7-34.

2. Ferlay J, Shin HR, Bray F, et al. Estimates of worldwide burden of cancer in 2008: GLOBOCAN 2008. Int J Cancer 2010;127:2893-917.

3. Macdonald JS. Gastric cancer: Nagoya is not New York. J 
Clin Oncol 2011;29:4348-50.

4. Crew KD, Neugut AI. Epidemiology of upper gastrointestinal malignancies. Semin Oncol 2004;31:450-64.

5. Devesa SS, Fraumeni JF Jr. The rising incidence of gastric cardia cancer. J Natl Cancer Inst 1999;91:747-9.

6. An international association between Helicobacter pylori infection and gastric cancer. The EUROGAST Study Group. Lancet 1993;341:1359-62.

7. Rubenstein JH, Taylor JB. Meta-analysis: the association of oesophageal adenocarcinoma with symptoms of gastro-oesophageal reflux. Aliment Pharmacol Ther 2010;32:1222-7.

8. Hampel H, Abraham NS, El-Serag HB. Meta-analysis: obesity and the risk for gastroesophageal reflux disease and its complications. Ann Intern Med 2005;143:199-211.

9. Cancer Genome Atlas Research N. Comprehensive molecular characterization of gastric adenocarcinoma. Nature 2014;513:202-9.

10. Cristescu R, Lee J, Nebozhyn M, et al. Molecular analysis of gastric cancer identifies subtypes associated with distinct clinical outcomes. Nat Med 2015;21:449-56.

11. Al-Batran SE, Hartmann JT, Probst S, et al. Phase III trial in metastatic gastroesophageal adenocarcinoma with fluorouracil, leucovorin plus either oxaliplatin or cisplatin: a study of the Arbeitsgemeinschaft Internistische Onkologie. J Clin Oncol 2008;26:1435-42.

12. Yoon HH, Bendell JC, Braiteh FS, et al. Ramucirumab combined with FOLFOX as front-line therapy for advanced esophageal, gastroesophageal junction, or gastric adenocarcinoma: a randomized, double-blind, multicenter Phase II trial. Ann Oncol 2016;27:2196-203.

13. Bang YJ, Van Cutsem E, Feyereislova A, et al. Trastuzumab in combination with chemotherapy versus chemotherapy alone for treatment of HER2-positive advanced gastric or gastro-oesophageal junction cancer (ToGA): a phase 3, open-label, randomised controlled trial. Lancet 2010;376:687-97.

14. Bartley AN, Washington MK, Colasacco C, et al. HER2 Testing and Clinical Decision Making in Gastroesophageal Adenocarcinoma: Guideline From the College of American Pathologists, American Society for Clinical Pathology, and the American Society of Clinical Oncology. J Clin Oncol 2017;35:446-64.

15. Wilke H, Muro K, Van Cutsem E, et al. Ramucirumab plus paclitaxel versus placebo plus paclitaxel in patients with previously treated advanced gastric or gastro-oesophageal junction adenocarcinoma (RAINBOW): a double-blind, randomised phase 3 trial. Lancet Oncol 2014;15:1224-35.

16. Li J, Qin S, Xu J, et al. Randomized, Double-Blind,
Placebo-Controlled Phase III Trial of Apatinib in Patients With Chemotherapy-Refractory Advanced or Metastatic Adenocarcinoma of the Stomach or Gastroesophageal Junction. J Clin Oncol 2016;34:1448-54.

17. Fuchs CS, Doi T, Jang RW, et al. Safety and Efficacy of Pembrolizumab Monotherapy in Patients With Previously Treated Advanced Gastric and Gastroesophageal Junction Cancer: Phase 2 Clinical KEYNOTE-059 Trial. JAMA Oncol 2018;4:e180013.

18. Kang YK, Boku N, Satoh T, et al. Nivolumab in patients with advanced gastric or gastro-oesophageal junction cancer refractory to, or intolerant of, at least two previous chemotherapy regimens (ONO-4538-12, ATTRACTION-2): a randomised, double-blind, placebocontrolled, phase 3 trial. Lancet 2017;390:2461-71.

19. Shitara K, Ozguroglu M, Bang YJ, et al. Pembrolizumab versus paclitaxel for previously treated, advanced gastric or gastro-oesophageal junction cancer (KEYNOTE-061): a randomised, open-label, controlled, phase 3 trial. Lancet 2018;392:123-33.

20. Tabernero J, Van Cutsem E, Bang YJ, et al., editors. Pembrolizumab with or without chemotherapy versus chemotherapy for advanced gastric or gastroesophageal junction (G/GEJ) adenocarcinoma: The phase III KEYNOTE-062 study.2019: J Clin Oncol 2019;37:abstr LBA4007.

21. Le DT, Uram JN, Wang H, et al. PD-1 Blockade in Tumors with Mismatch-Repair Deficiency. N Engl J Med 2015;372:2509-20.

22. Al-Batran SE, Homann N, Pauligk C, et al. Perioperative chemotherapy with fluorouracil plus leucovorin, oxaliplatin, and docetaxel versus fluorouracil or capecitabine plus cisplatin and epirubicin for locally advanced, resectable gastric or gastro-oesophageal junction adenocarcinoma (FLOT4): a randomised, phase 2/3 trial. Lancet 2019;393:1948-57.

23. Bang YJ, Kim YW, Yang HK, et al. Adjuvant capecitabine and oxaliplatin for gastric cancer after D2 gastrectomy (CLASSIC): a phase 3 open-label, randomised controlled trial. Lancet;379:315-21.

24. Yoshida K, Kodera Y, Kochi M, et al. Addition of Docetaxel to Oral Fluoropyrimidine Improves Efficacy in Patients With Stage III Gastric Cancer: Interim Analysis of JACCRO GC-07, a Randomized Controlled Trial. J Clin Oncol 2019;37:1296-304.

25. Smyth EC, Wotherspoon A, Peckitt C, et al. Mismatch Repair Deficiency, Microsatellite Instability, and Survival: An Exploratory Analysis of the Medical Research Council Adjuvant Gastric Infusional Chemotherapy (MAGIC) 
Trial. JAMA Oncol 2017;3:1197-203.

26. Choi YY, Kim H, Shin SJ, et al. Microsatellite Instability and Programmed Cell Death-Ligand 1 Expression in Stage II/III Gastric Cancer: Post Hoc Analysis of the CLASSIC Randomized Controlled study. Ann Surg 2019;270:309-16.

27. Pietrantonio F, Miceli R, Raimondi A, et al. Individual Patient Data Meta-Analysis of the Value of Microsatellite Instability As a Biomarker in Gastric Cancer. J Clin Oncol 2019;37:3392-400.

28. Cheng DT, Mitchell TN, Zehir A, et al. Memorial Sloan Kettering-Integrated Mutation Profiling of Actionable Cancer Targets (MSK-IMPACT): A Hybridization Capture-Based Next-Generation Sequencing Clinical Assay for Solid Tumor Molecular Oncology. J Mol Diagn 2015;17:251-64.

29. Niu B, Ye K, Zhang Q, et al. MSIsensor: microsatellite instability detection using paired tumor-normal sequence data. Bioinformatics 2014;30:1015-6.

30. Ptashkin RN, Mandelker DL, Coombs CC, et al. Prevalence of Clonal Hematopoiesis Mutations in TumorOnly Clinical Genomic Profiling of Solid Tumors. JAMA Oncol 2018;4:1589-93.

31. Meric-Bernstam F, Brusco L, Shaw K, et al. Feasibility of Large-Scale Genomic Testing to Facilitate Enrollment Onto Genomically Matched Clinical Trials. J Clin Oncol 2015;33:2753-62.

32. Greally M, Walch H, Chou J, et al., editors. Clinical and molecular factors predicting resistance to first-line (1L) FOLFOX in patients (pts) with advanced esophagogastric cancer (EGA) and patterns of subsequent therapy.2019: J Clin Oncol 2019;37:abstr 4047.

33. Janjigian YY, Sanchez-Vega F, Jonsson P, et al. Genetic Predictors of Response to Systemic Therapy in Esophagogastric Cancer. Cancer Discov 2018;8:49-58.

34. Fuchs CS, Tabernero J, Tomasek J, et al. Biomarker analyses in REGARD gastric/GEJ carcinoma patients treated with VEGFR2-targeted antibody ramucirumab. $\mathrm{Br}$ J Cancer 2016;115:974-82.

35. Van Cutsem E, de Haas S, Kang YK, et al. Bevacizumab in combination with chemotherapy as first-line therapy in advanced gastric cancer: a biomarker evaluation from the AVAGAST randomized phase III trial. J Clin Oncol 2012;30:2119-27.

36. Greally M, Chou JF, Chatila WK, et al. Clinical and Molecular Predictors of Response to Immune Checkpoint Inhibitors in Patients with Advanced Esophagogastric Cancer. Clin Cancer Res 2019;25:6160-9.

37. Lordick F, Kang YK, Chung HC, et al. Capecitabine and cisplatin with or without cetuximab for patients with previously untreated advanced gastric cancer (EXPAND): a randomised, open-label phase 3 trial. Lancet Oncol 2013;14:490-9.

38. Waddell T, Chau I, Cunningham D, et al. Epirubicin, oxaliplatin, and capecitabine with or without panitumumab for patients with previously untreated advanced oesophagogastric cancer (REAL3): a randomised, openlabel phase 3 trial. Lancet Oncol 2013;14:481-9.

39. Maron SB, Alpert L, Kwak HA, et al. Targeted Therapies for Targeted Populations: Anti-EGFR Treatment for EGFR-Amplified Gastroesophageal Adenocarcinoma. Cancer Discov 2018;8:696-713.

40. Golan T, Hammel P, Reni M, et al. Maintenance Olaparib for Germline BRCA-Mutated Metastatic Pancreatic Cancer. N Engl J Med 2019;381:317-27.

41. Bang YJ, Im SA, Lee KW, et al. Randomized, DoubleBlind Phase II Trial With Prospective Classification by ATM Protein Level to Evaluate the Efficacy and Tolerability of Olaparib Plus Paclitaxel in Patients With Recurrent or Metastatic Gastric Cancer. J Clin Oncol 2015;33:3858-65.

42. Bang YJ, Xu RH, Chin K, et al. Olaparib in combination with paclitaxel in patients with advanced gastric cancer who have progressed following first-line therapy (GOLD): a double-blind, randomised, placebo-controlled, phase 3 trial. Lancet Oncol 2017;18:1637-51.

43. Lowery MA, Kelsen DP, Capanu M, et al. Phase II trial of veliparib in patients with previously treated BRCAmutated pancreas ductal adenocarcinoma. Eur J Cancer 2018;89:19-26.

44. Van Cutsem E, Bang YJ, Mansoor W, et al. A randomized, open-label study of the efficacy and safety of AZD4547 monotherapy versus paclitaxel for the treatment of advanced gastric adenocarcinoma with FGFR2 polysomy or gene amplification. Ann Oncol 2017;28:1316-24.

45. Kuboki Y, Matsubara N, Bando H, et al. editors. First-inhuman (FIH) study of TAS-120, a highly selective covalent oral fibroblast growth factor receptor (FGFR) inhibitor, in patients (pts) with advanced solid tumors. Ann Oncol 2017;28:v122-41.

46. Corcoran RB, Chabner BA. Application of Cell-free DNA Analysis to Cancer Treatment. N Engl J Med 2018;379:1754-65.

doi: $10.21037 /$ tgh.2020.01.09

Cite this article as: Ku GY. Next generation sequencing in gastric or gastroesophageal adenocarcinoma. Transl Gastroenterol Hepatol 2020;5:56. 